\title{
Variations in COVID-19 vaccination uptake among people in receipt of psychotropic drugs: cross- sectional analysis of a national population-based prospective cohort
}

Siobhán Murphy*, Dermot O’Reilly*, Rhiannon K. Owen, Ashley Akbari, Emily Lowthian, Stuart Bedston, Fatemeh Torabi, Jillian Beggs, Antony Chuter, Simon de Lusignan, Richard Hobbs, Chris Robertson, Srinivasa Vittal Katikireddi, Aziz Sheikh and Declan T. Bradley

\section{Background}

Coronavirus disease 2019 (COVID-19) has disproportionately affected people with mental health conditions.

\section{Aims}

We investigated the association between receiving psychotropic drugs, as an indicator of mental health conditions, and COVID-19 vaccine uptake.

\section{Method}

We conducted a cross-sectional analysis of a prospective cohort of the Northern Ireland adult population using national linked primary care registration, vaccination, secondary care and pharmacy dispensing data. Univariable and multivariable logistic regression analyses investigated the association between anxiolytic, antidepressant, antipsychotic, and hypnotic use and COVID-19 vaccination status, accounting for age, gender, deprivation and comorbidities. Receiving any COVID-19 vaccine was the primary outcome.

\section{Results}

There were 1433814 individuals, of whom 1166917 received a COVID-19 vaccination. Psychotropic medications were dispensed to 267049 people. In univariable analysis, people who received any psychotropic medication had greater odds of receiving COVID-19 vaccination: odds ratio (OR) = 1.42 (95\% Cl 1.41-1.44).
However, after adjustment, psychotropic medication use was associated with reduced odds of vaccination $\left(\mathrm{OR}_{\mathrm{adj}}=0.90\right.$ $95 \% \mathrm{Cl} 0.89-0.91)$. People who received anxiolytics $\left(\mathrm{OR}_{\mathrm{adj}}=0.63\right.$, $95 \% \mathrm{Cl} 0.61-0.65)$, antipsychotics ( $\left.\mathrm{OR}_{\mathrm{adj}}=0.75,95 \% \mathrm{Cl} 0.73-0.78\right)$ and hypnotics $\left(\mathrm{OR}_{\mathrm{adj}}=0.90,95 \% \mathrm{Cl} 0.87-0.93\right)$ had reduced odds of being vaccinated. Antidepressant use was not associated with vaccination $\left(\mathrm{OR}_{\mathrm{adj}}=1.02,95 \% \mathrm{Cl} 1.00-1.03\right)$

\section{Conclusions}

We found significantly lower odds of vaccination in people who were receiving treatment with anxiolytic and antipsychotic medications. There is an urgent need for evidence-based, tailored vaccine support for people with mental health conditions.

\section{Keywords}

Covid-19; vaccines; mental health; equity; psychotropic medicines.

\section{Copyright and usage}

(c) The Author(s), 2022. Published by Cambridge University Press. This is an Open Access article, distributed under the terms of the Creative Commons Attribution licence (https://creativecommons.org/licenses/by/4.0/), which permits unrestricted re-use, distribution, and reproduction in any medium, provided the original work is properly cited

\section{Background}

The coronavirus disease 2019 (COVID-19) pandemic, caused by the novel coronavirus, SARS-CoV-2, is a global disaster that has highlighted and exacerbated health inequalities. ${ }^{1}$ The pandemic caused depression and anxiety; non-pharmaceutical interventions led to isolation; societal disruption affected job security; and mental health services were disrupted globally., ${ }^{2,3}$ A number of vaccines against COVID-19 have been rapidly developed and delivered. As of October 2021, four vaccines, the BNT162b2 mRNA (PfizerBioNTech), ChAdOx1 nCoV-19 adenoviral (OxfordAstraZeneca), mRNA-1273 (Moderna) and Ad26.COV2-S (Janssen) vaccines have been approved by the UK Medicines and Healthcare products Regulatory Agency. The COVID-19 vaccination programme in the UK began on 8 December 2020, with priority given to older age groups, residents and staff in care homes, healthcare workers, people who are clinically extremely vulnerable, and people with informal caring responsibilities. Subsequent delivery among the general population was prioritised from older to younger age groups and towards those with specific clinical conditions. The effectiveness of the vaccination strategy in controlling

* Joint first authors. transmission depends on high uptake levels in the population. High levels of vaccine hesitancy have been documented in certain groups such as young adults, females, people from ethnic minorities and those in low-income households. ${ }^{4,5}$

People who have mental illness, including schizophrenia, bipolar disorder, schizoaffective disorder and major depressive disorder, are at increased risk of COVID-19 infection and experience higher rates of COVID-19 hospital admission and death. ${ }^{6,7}$ People who have mental illness have higher levels of non-communicable diseases such as cardiovascular and respiratory diseases, diabetes and cancer ${ }^{8}$ resulting in a life expectancy 15-20 years shorter than their peers. ${ }^{9}$ This situation arises from a complex interplay of biological, psychological and social factors, which may be partly mediated by a higher prevalence of hazardous health behaviours such as smoking, and not engaging in health protective behaviours, including health screening. ${ }^{10,11}$ For these reasons, the UK's Joint Committee on Vaccinations and Immunisation identified people with mental illness as a priority for COVID-19 vaccination, as did, for example, the governments of Denmark, Germany and the Netherlands. ${ }^{12}$ There have been mixed reports about uptake of COVID-19 vaccination in those with poor mental health. ${ }^{4,13,14}$ One Danish study reported only 
slightly lower vaccine willingness among people with mental illness than among the wider population. ${ }^{13}$

\section{Aims}

To our knowledge, no previous study has examined COVID-19 vaccination uptake at a national level among people who have mental health conditions. It is important to understand the relationship between psychiatric morbidity and uptake of COVID-19 vaccination to understand the equity of service provision and to evaluate the effects of current approaches for reaching people who have mental health conditions. Northern Ireland has higher rates of mental health conditions compared with other UK nations, with rates 25\% higher than England. ${ }^{15}$ As of October 2021, Northern Ireland also has the lowest COVID-19 vaccination uptake of the four UK nations according to government reporting. ${ }^{16}$ We aimed to investigate the association between mental health conditions (overall and individually) and vaccination uptake in the adult population. We used primary care dispensing of psychotropic drugs as an indicator of mental health conditions. We explored whether mental health conditions explained the lower uptake of COVID-19 vaccination among people who live in deprived areas.

\section{Method}

\section{Data sources}

We constructed a national cohort using a unique data-set consisting of national linked primary care registration, vaccination and pharmacy dispensing data. We used information on vaccination from the Vaccine Management System (VMS), linked to population data from the National Health Authority Information System (NHAIS, recording eligibility for healthcare in Northern Ireland), and data on medications dispensed by community pharmacists from the Enhanced Prescribing Database. We used emergency department attendance data and secondary care Patient Administration System data as part of the process to identify whether a person was resident in Northern Ireland. The Patient Administration System data included all acute hospital sites in Northern Ireland, but not all mental health and intellectual disability (also known as learning disability in UK heathcare) settings. Primary care consultation and diagnostic data were not available for the project. All data were linked by the unique identifier and then an anonymous data-set was made available to the research team through the Business Services Organisation's online Secure Research Platform (Supplementary Figure 1).

The cohort was defined as people aged 18 and over on 1 December 2020 and identified the vaccine status for those still alive in Northern Ireland on 9 September 2021, which was the final day of data provision. The project was approved by the Honest Broker Service Governance Board, project number 064 . The study used fully anonymised data and therefore participant consent was not required.

\section{Study population}

Northern Ireland has a universal, free at the point-of-service healthcare system with eligibility based on registration in NHAIS. This register comprises the entire population registered with a GP and, for each patient, holds basic demographic information relating to age, gender and current address, as well as a unique identifier (the health and care number), which can be used for linkage across health-related data-sets. NHAIS also receives regular updates from the General Register Office on the date and cause of death, which adds to the reliability of the data. The study cohort was based on all registered individuals aged 18 years and over on 1 December 2020 and living in Northern Ireland. Residents in institutional dwellings such as care homes $(n=14547)$ were not included in the study as they were subject to specific targeted interventions and uptake is recognised to be uniformly high. ${ }^{17}$ Identifying such residents is recognised to be difficult ${ }^{18}$ but here we used any of:

(a) an indicator on NHAIS related to care home payment;

(b) residence at a property with ten or more residents with a mean age of $>65$ years;

(c) being aged over 70 and having more than ten reverse transcription polymerase chain reaction (RT-PCR) tests before 8 December 2020 (a proxy for participation in a care home mass testing programme); or

(d) aged over 70 and receiving the BNT162b2 vaccine before 1 February 2021, as during this period it was only available to health and social care workers and care home residents.

Other cohort attributes were derived from the household and address information in NHAIS. The unique property reference number (UPRN), which identifies individual residences such as houses and flats, was used as a proxy for households and thus to identify the number of people per household (categorised into single-person households, two-person, three or four and five or more person households). Socioeconomic status was also based on address; the UPRN was used to assign the property value used by central government to determine the level of local tax payable by each household. Property values were categorised as $(<£ 100000$; $£ 100000-£ 124999$; $£ 125000-£ 199$ 999; $£ 200$ 000-249 999; $£ 250000+$ ). Individuals were also allocated, based on their area of residence, to quintiles of deprivation using the Northern Ireland Multiple Deprivation Measure 2017. ${ }^{19}$

\section{Health status}

Given the extant literature of the co-occurrence of mental and physical health problems ${ }^{8}$ we included a measure of multimorbidity. This measure was based on a count of the number of different British National Formulary (BNF) chapters the patient had been receiving drugs from. To be included in the count, a medicine had to be prescribed in both of two 3-month periods in the 6 months before the vaccination programme was implemented. This method was adapted from one validated in other multimorbidity studies using administrative data. ${ }^{20}$ Medications related to contraceptives (BNF chapter 7, section 3) were removed as these do not indicate an illness, and medicines related to psychiatric morbidity (BNF chapter 4 sections 1, 2 and 3) were removed from this overall measure so that they could be investigated separately.

\section{Exposure definition}

We used prescriptions to create four variables; hypnotics (BNF chapter 4.1.1) anxiolytics (BNF chapter 4.1.2), antipsychotics (BNF chapter 4.2) and antidepressants (BNF chapter 4.3). As with the general health status indicator, the medicine had to be prescribed in both 3-month periods before the start of the vaccination period to be counted. We selected the period of time before the start of the vaccination programme to avoid the possibility of any causal relationship in the opposite direction, such as one in which prescription of psychotropic medication was influenced by vaccine status.

\section{Outcome definition}

The vaccination status of patients was derived from the VMS and defined as having at least one dose of a COVID-19 vaccine up to and including 9 September 2021. The VMS collates information from any setting where vaccination is delivered - GP practice, clinic, care home, patient's home (housebound), ward (long-stay patients) and community pharmacy, the date, dose and type of all vaccines administered. 


\section{Analytic approach}

Administrative data can contain individuals who are no longer part of the study population because it is not possible to elicit the fact of their having left a region from the administrative data. Following the approach described in Vasileiou et $\mathrm{al}^{21}{ }^{21}$ we assigned a weight of one to every individual who was identified as having on or after 1 January 2020 any hospital admission, emergency department attendance, any prescription dispensed, a COVID-19 test taken, or a COVID-19 vaccine administered. Individuals who had no contact with any of these healthcare services were weighted such that the sum of weights for their combined gender and 5-year age band was equal to the NISRA 2020 mid-year population estimate. This resulted in an individual weight for each record between zero and one.

We agreed a statistical analysis plan before commencing the analysis. The statistical analysis code is available on the DaCVaP GitHub repository (https://github.com/HDRUK/DaCVaP). We followed the Reporting of studies Conducted using Observational Routinely-collected Data checklist to guide transparent reporting of this cohort study.

We report results for the entire population over 18 years of age, and a subgroup analysis for the younger age groups (those aged 1839 years), chosen a priori with the aim of understanding whether the same risk factors were associated with vaccination in younger age group, who had less time to get vaccinated. We described the cohort with weighted counts and percentages by strata.

We conducted logistic regression analyses with binary vaccination status that included all vaccinations up to and including 9 September 2021 as the dependent variable. The models were first adjusted for demographic, socioeconomic and health indicators with mental health prescriptions added to the fully adjusted models. Logistic regression for the vaccine status in a cross-sectional study design was used in preference to Cox proportional hazards in a longitudinal design because the date of eligibility for vaccination is difficult to define at an individual level for comorbidities, age groups, occupation, health and caregiver status in a longitudinal design. All adult age groups were eligible for vaccination in Northern Ireland at the time of our study.

Our primary outcome was any first vaccination; this included Pfizer-BioNTech, Oxford-AstraZeneca and Moderna vaccines as during the study period Janssen was not administered in Northern Ireland. Secondary outcomes were second-dose vaccination, compared with the single-dose-only population, and the unvaccinated population. As a supplementary analysis to investigate how sociodemographic factors and comorbidities influenced dispensing of psychotropic medications, we performed univariable and multivariable logistic regression with each of anxiolytic, hypnotic, antidepressant and antipsychotic use as binary dependent variables.

Weighting was necessary for the regression because the 'null' records for people who were no longer truly present in the region would otherwise introduce bias: they were by definition unvaccinated and had no contact with healthcare services, including receiving no medications. Failing to correct for this would increase the relative odds of medication use in the vaccinated group. Downweighting them reduces this bias by reducing the overall unvaccinated subgroup who have no healthcare contacts to the size estimated in the age- and gender-specific population estimate. We used $R$ version 4.1.0 and $\mathrm{glm}$ using the weights option for univariable and multivariable binary logistic regression with the weights described above, with Wald 95\% confidence limits.

\section{Role of the funding source}

The sponsor of the study had no role in study design, data analysis, data interpretation, writing of the report, or the decision to submit the paper for publication.

\section{Results}

The cohort consisted of 1433814 individuals, of which 267049 (19\%) individuals had received psychotropic medication in both serial 3-month periods before the vaccination programme started. Antidepressants, used by $21 \%$ of women and $12 \%$ of men, were the most common medication, and antipsychotic medication used by $2 \%$ of the adult population was the least common; and around $3 \%$ had been prescribed hypnotics and anxiolytics.

Table 1 (and Supplementary Table 1 available at https://doi.org/ 10.1192/bjp.2022.36) shows the cohort characteristics on each of the four different types of medication. The use of psychotropic medication increased with age. Women were more likely to be prescribed anxiolytics, hypnotics and antidepressants than men; in the fully adjusted models, men were more likely to be prescribed antipsychotics than women (odds ratio $(\mathrm{OR})=1.51,95 \%$ CI 1.47-1.55; Supplementary Table 2). Strong deprivation gradients were evident across all four mental health categories, which were attenuated upon adjusting for other characteristics.

There were substantial associations between the extent of physical ill health (as measured by the number of prescription categories dispensed in the previous 6 months) and psychotropic medication use, especially for antidepressants (OR $=19.01,95 \%$ CI 18.54-19.49) for those on five different non-psychotropic medications compared with those on none). Comorbidity was common among mental health categories with the strongest association between antidepressants and antipsychotics, even after adjusting for other factors.

As of 9 September 2021, 1166917 adults had received at least one first dose vaccination in Northern Ireland $(81 \%$ of the adult population). Coverage was over $90 \%$ for people aged more than 60 years and $64 \%$ for those aged 18-29. The odds of having at least one vaccination was lower in males $\left(\mathrm{OR}_{\mathrm{adj}}=0.92,95 \% \mathrm{CI}\right.$ 0.92-0.93).

People who lived in the greatest value houses or least deprived areas had greater odds of vaccination $\left(\mathrm{OR}_{\mathrm{adj}}=2.12,95 \%\right.$ CI 2.06-2.17, and $\mathrm{OR}_{\mathrm{adj}}=1.54,95 \%$ CI 1.51-1.57) in fully adjusted models. Approximately $90 \%$ of people receiving medications for two or more physical conditions had been vaccinated with at least one dose, whereas $75 \%$ of those on no such medications were vaccinated. In a univariable analysis, people on any psychotropic medication were more likely to be vaccinated than those who were not on such medications (OR $=1.42,95 \%$ CI 1.41-1.44), however, when adjusted for demographic, socioeconomic factors and multimorbidity, having psychotropic medications was associated with a significantly reduced odds of vaccination $\left(\mathrm{OR}_{\mathrm{adj}}=0.9095 \% \mathrm{CI} 0.89-0.91\right)$.

Table 2 shows the odds of being vaccinated (with at least one dose) according to the type of psychotropic medication prescribed. In the unadjusted models, both antidepressants and hypnotics were associated with increased odds of being vaccinated; however, these were markedly reduced with adjustment for sociodemographic, socioeconomic and physical health factors. In the fully adjusted models, individuals in receipt of anxiolytics $\left(\mathrm{OR}_{\mathrm{adj}}=0.63,95 \% \mathrm{CI}\right.$ $0.61-0.65)$, on antipsychotics $\left(\mathrm{OR}_{\mathrm{adj}}=0.75,95 \%\right.$ CI $\left.0.73-0.78\right)$ and hypnotics $\left(\mathrm{OR}_{\mathrm{adj}}=0.90,95 \% \mathrm{CI} 0.87-0.93\right)$ had reduced odds of COVID-19 vaccination. Antidepressant use was not associated with vaccination $\left(\mathrm{OR}_{\mathrm{adj}}=1.02,95 \% \mathrm{CI} 1.00-1.03\right)$.

We completed analyses on second-dose vaccinations compared with unvaccinated and second dose compared with first dose only as secondary outcomes (Supplementary Table 3). The results revealed similar trends with those in receipt of anxiolytics, antipsychotics and hypnotics having a lower likelihood of vaccine uptake and there were negligible differences for those in receipt of antidepressants.

The difference between the adjusted and the fully adjusted models in Table 2 shows that controlling for mental health had 
Table 1 Variation in use of psychotropic medication according to type of medication ${ }^{a}$ [Typesetter: In both tables please align data by adding thin spaces to numbers over 1000 as per house style for tables and ensure all alignment is then correct.]

\begin{tabular}{|c|c|c|c|c|}
\hline Variable & Anxiolytics, $n(\%)$ & Hypnotics, $n$ (\%) & Antidepressants, $n(\%)$ & Antipsychotics, $n(\%)$ \\
\hline \multicolumn{5}{|l|}{ Gender } \\
\hline Female & $26072(3.6)$ & $26816(3.7)$ & $153004(20.9)$ & $15266(2.1)$ \\
\hline Male & $13893(2.0)$ & $15872(2.3)$ & 83425 (11.9) & $14791(2.1)$ \\
\hline \multicolumn{5}{|l|}{ Age bands, years } \\
\hline $18-29$ & 2901 (4.2) & $6251(9.0)$ & $11464(16.4)$ & $1030(1.5)$ \\
\hline $30-39$ & $5450(3.9)$ & $8484(6.1)$ & $26222(18.7)$ & $2504(1.8)$ \\
\hline $40-49$ & 7537 (3.8) & $9112(4.6)$ & $45715(23.0)$ & $5285(2.7)$ \\
\hline $50-59$ & $9347(3.6)$ & 8959 (3.5) & $59044(23.0)$ & $7586(2.9)$ \\
\hline $60-69$ & $7362(3.0)$ & $5204(2.2)$ & $43693(18.1)$ & $6058(2.5)$ \\
\hline 70-79 & $5167(2.1)$ & $2888(1.2)$ & 31136 (12.4) & $4605(1.8)$ \\
\hline$\geq 80$ & $2201(0.8)$ & $1790(0.6)$ & $19155(6.9)$ & 2989 (1.1) \\
\hline \multicolumn{5}{|l|}{ Household } \\
\hline 1 & $9634(5.6)$ & $11532(6.7)$ & $42277(24.7)$ & $8041(4.7)$ \\
\hline 2 & $10674(3.4)$ & $12785(4.1)$ & $60243(19.2)$ & 7096 (2.3) \\
\hline $3-4$ & $12908(2.2)$ & $12300(2.1)$ & $89348(15.2)$ & $9299(1.6)$ \\
\hline$\geq 5$ & $2880(1.9)$ & $2466(1.6)$ & $18076(11.8)$ & $2452(1.6)$ \\
\hline Missing & $3869(1.9)$ & 3605 (1.7) & $26485(12.8)$ & $3169(1.5)$ \\
\hline \multicolumn{5}{|l|}{ Deprivation } \\
\hline Most deprived & $11878(4.3)$ & $10891(3.9)$ & $60174(21.7)$ & 9768 (3.5) \\
\hline 2 & $9127(3.1)$ & $10210(3.5)$ & $51788(17.7)$ & $7012(2.4)$ \\
\hline 3 & $7393(2.5)$ & $8529(2.9)$ & $46123(15.7)$ & $5616(1.9)$ \\
\hline 4 & $6807(2.3)$ & 7235 (2.4) & $42763(14.4)$ & $4547(1.5)$ \\
\hline Least deprived & $4760(1.7)$ & $5823(2.1)$ & 35581 (13.0) & $3114(1.1)$ \\
\hline \multicolumn{5}{|l|}{ House value } \\
\hline$<74999$ & $13451(5.1)$ & $13381(5.1)$ & $65084(24.8)$ & $10756(4.1)$ \\
\hline 75 000-99999 & $10618(3.6)$ & $10389(3.5)$ & $57954(19.7)$ & 7451 (2.5) \\
\hline 100 000-124999 & $5691(2.4)$ & $5966(2.6)$ & $36877(15.9)$ & $3911(1.7)$ \\
\hline 125 000-199999 & $6443(1.7)$ & 8105 (2.1) & $49832(13.0)$ & 4438 (1.2) \\
\hline $200000-249999$ & $1227(1.2)$ & $1637(1.6)$ & $10448(10.1)$ & $838(0.8)$ \\
\hline$>250000$ & $927(0.9)$ & $1482(1.5)$ & 8237 (8.4) & $674(0.7)$ \\
\hline Missing & $1608(2.8)$ & 1728 (3.0) & 7997 (13.7) & 1989 (3.4) \\
\hline \multicolumn{5}{|l|}{ Settlement band } \\
\hline Urban & 10426 (3.6) & 9062 (3.1) & $54972(19.1)$ & $8058(2.8)$ \\
\hline Intermediate & $19508(2.9)$ & 21068 (3.2) & $116372(17.4)$ & $14682(2.2)$ \\
\hline Rural & $10031(2.1)$ & $12558(2.6)$ & $65085(13.6)$ & 7317 (1.5) \\
\hline \multicolumn{5}{|c|}{ Physical multimorbidity } \\
\hline 0 & $3975(0.5)$ & $3698(0.5)$ & $49036(6.1)$ & $5174(0.6)$ \\
\hline 1 & $6711(2.7)$ & $6510(2.6)$ & 48285 (19.4) & 5747 (2.3) \\
\hline 2 & $7403(4.7)$ & $7857(5.0)$ & $43370(27.5)$ & 5567 (3.5) \\
\hline 3 & 7526 (7.4) & $8220(8.0)$ & $37866(37.0)$ & $5021(4.9)$ \\
\hline 4 & $6189(9.9)$ & 7090 (11.3) & 27825 (44.5) & 3957 (6.3) \\
\hline 5 & $8161(14.9)$ & $9313(17.0)$ & 30047 (54.7) & $4591(8.4)$ \\
\hline \multicolumn{5}{|l|}{ Anxiolytics } \\
\hline No & - & 30576 (2.2) & $208112(14.9)$ & $21646(1.6)$ \\
\hline Yes & $39965(100.0)$ & 12112 (30.3) & $28317(70.9)$ & 8411 (21.0) \\
\hline \multicolumn{5}{|l|}{ Hypnotics } \\
\hline No & $27853(2.0)$ & & 209609 (15.1) & $22514(1.6)$ \\
\hline Yes & $12112(28.4)$ & $42688(100.0)$ & $26820(62.8)$ & 7543 (17.7) \\
\hline \multicolumn{5}{|l|}{ Antidepressants } \\
\hline No & $11648(1.0)$ & $15868(1.3)$ & - & $7789(0.7)$ \\
\hline Yes & 28317 (12.0) & 26820 (11.3) & $236429(100.0)$ & 22268 (9.4) \\
\hline \multicolumn{5}{|l|}{ Antipsychotics } \\
\hline No & 31554 (2.2) & 35145 (2.5) & 214161 (15.3) & - \\
\hline Yes & 8411 (28.0) & 7543 (25.1) & $22268(74.1)$ & 30057 (100.0) \\
\hline
\end{tabular}

only marginal effects on the vaccination uptake among people who were deprived (by area or house value). Before adjustment for psychotropic medication use there was a twofold difference in odds of vaccination between those living in the more expensive compared with less expensive housing $\left(\mathrm{OR}_{\mathrm{adj}}=2.14,95 \% \mathrm{CI} 2.08-2.20\right)$ and a marked difference between the least and most deprivation quintiles $\left(\mathrm{OR}_{\mathrm{adj}}=1.55,95 \% \mathrm{CI} 1.52-1.58\right)$; adjustment for psychotropic medications had virtually no effect on these socioeconomic gradients.

Supplementary Table 4 presents the unadjusted and adjusted ORs for vaccine uptake in those under 40 years of age. There was a similar trend in the under 40 population with variations across different types of mental health issues. Antidepressants were associated with increased odds of vaccination in the unadjusted and fully adjusted models, and all other types of psychotropic medications were associated with decreased odds of vaccination. In the fully adjusted analyses, the use of anxiolytics was associated with reduced odds of vaccination $\left(\mathrm{OR}_{\mathrm{adj}}=0.57,95 \%\right.$ CI $\left.0.53-0.60\right)$. Individuals who were prescribed antipsychotics $\left(\mathrm{OR}_{\mathrm{adj}}=0.75\right.$, 95\% CI 0.71-0.80) and hypnotics $\left(\mathrm{OR}_{\mathrm{adj}}=0.78,95 \%\right.$ CI $\left.0.72-0.84\right)$ had lower odds of vaccination compared with those who did not receive those medicines. 
Table 2 Unadjusted and adjusted odds ratios for examining variation (weighted) in full cohort ${ }^{\mathrm{a}}$

\begin{tabular}{|c|c|c|c|c|c|}
\hline & Cohort, $n$ (column \%) & Vaccinated, $n$ (row \%) & Unadjusted OR $(95 \% \mathrm{Cl})$ & Adjusted $(95 \% \mathrm{Cl})$ & Fully adjusted $(95 \% \mathrm{Cl})$ \\
\hline \multicolumn{6}{|l|}{ Gender } \\
\hline Female & 732504 (51.1) & $603293(82.4)$ & & & \\
\hline Male & 701310 (48.9) & $563624(80.4)$ & $0.88(0.87-0.88)$ & $0.93(0.92-0.94)$ & $0.92(0.92-0.93)$ \\
\hline \multicolumn{6}{|l|}{ Age bands, years } \\
\hline$\geq 80$ & $69745(4.9)$ & $63263(90.7)$ & & & \\
\hline 70-79 & $139927(9.8)$ & 131327 (93.9) & $1.56(1.51-1.62)$ & $1.56(1.51-1.62)$ & $1.58(1.52-1.64)$ \\
\hline $60-69$ & $198712(13.9)$ & $184363(92.8)$ & $1.32(1.28-1.36)$ & $1.43(1.38-1.48)$ & $1.47(1.42-1.51)$ \\
\hline $50-59$ & 257166 (17.9) & $228744(88.9)$ & $0.82(0.80-0.85)$ & $0.97(0.94-1.00)$ & $1.01(0.98-1.04)$ \\
\hline $40-49$ & $242023(16.9)$ & 201523 (83.3) & $0.51(0.50-0.52)$ & $0.66(0.64-0.68)$ & $0.69(0.67-0.71)$ \\
\hline $30-39$ & 250532 (17.5) & $181014(72.3)$ & $0.27(0.26-0.27)$ & $0.39(0.37-0.40)$ & $0.40(0.39-0.41)$ \\
\hline $18-29$ & $275708(19.2)$ & $176683(64.1)$ & $0.18(0.18-0.19)$ & $0.26(0.26-0.27)$ & $0.27(0.26-0.28)$ \\
\hline \multicolumn{6}{|l|}{ Household } \\
\hline 1 & $171428(12.0)$ & $144490(84.3)$ & & & \\
\hline 2 & 314104 (21.9) & 270818 (86.2) & $1.17(1.15-1.19)$ & $1.12(1.10-1.14)$ & $1.11(1.09-1.13)$ \\
\hline $3-4$ & 587909 (41.0) & 481910 (82.0) & $0.85(0.84-0.86)$ & $1.17(1.15-1.19)$ & $1.15(1.13-1.17)$ \\
\hline $5+$ & $153372(10.7)$ & $107976(70.4)$ & $0.44(0.44-0.45)$ & $0.73(0.72-0.75)$ & $0.72(0.70-0.73)$ \\
\hline Missing & 207001 (14.4) & $161723(78.1)$ & & & \\
\hline \multicolumn{6}{|l|}{ Deprivation } \\
\hline Most deprived & 276734 (19.3) & $205196(74.1)$ & & & \\
\hline 2 & $293043(20.4)$ & $232596(79.4)$ & $1.34(1.33-1.36)$ & $1.12(1.10-1.13)$ & $1.11(1.10-1.13)$ \\
\hline 3 & $293343(20.5)$ & 240691 (82.1) & $1.59(1.57-1.61)$ & $1.22(1.20-1.24)$ & $1.21(1.19-1.23)$ \\
\hline 4 & $297232(20.7)$ & $249682(84.0)$ & $1.83(1.81-1.85)$ & $1.30(1.28-1.32)$ & $1.29(1.27-1.32)$ \\
\hline Least deprived & 273462 (19.1) & 238752 (87.3) & $2.40(2.36-2.43)$ & $1.55(1.52-1.58)$ & $1.54(1.51-1.57)$ \\
\hline \multicolumn{6}{|l|}{ House value } \\
\hline$<74999$ & 262813 (18.3) & $198538(75.5)$ & & & \\
\hline 75 000-99999 & 294879 (20.6) & 229647 (77.9) & $1.14(1.13-1.15)$ & $1.22(1.20-1.24)$ & $1.21(1.20-1.23)$ \\
\hline 100-124999 & 232607 (16.2) & $189820(81.6)$ & $1.44(1.42-1.46)$ & $1.46(1.44-1.48)$ & $1.45(1.42-1.47)$ \\
\hline 125-199999 & 384088 (26.8) & 328731 (85.6) & $1.92(1.90-1.95)$ & $1.82(1.79-1.84)$ & $1.80(1.77-1.83)$ \\
\hline 200-249999 & $103176(7.2)$ & 90218 (87.4) & $2.25(2.21-2.30)$ & $2.11(2.05-2.16)$ & $2.08(2.03-2.14)$ \\
\hline$>250000$ & $97852(6.8)$ & $86164(88.1)$ & $2.39(2.34-2.44)$ & $2.14(2.08-2.20)$ & $2.12(2.06-2.17)$ \\
\hline Missing & $58399(4.1)$ & $43799(75.0)$ & & & \\
\hline \multicolumn{6}{|l|}{ Settlement band } \\
\hline Urban & $288136(20.1)$ & $223796(77.7)$ & & & \\
\hline Intermediate & $668530(46.6)$ & 544803 (81.5) & $1.27(1.25-1.28)$ & $1.04(1.03-1.06)$ & $1.04(1.03-1.06)$ \\
\hline Rural & 477147 (33.3) & 398318 (83.5) & $1.45(1.44-1.47)$ & $1.12(1.11-1.14)$ & $1.12(1.10-1.14)$ \\
\hline \multicolumn{6}{|l|}{ Prescriptions } \\
\hline 0 & $807784(56.3)$ & $608279(75.3)$ & & & \\
\hline 1 & 248418 (17.3) & $215180(86.6)$ & $2.12(2.1-2.15)$ & $1.4(1.38-1.42)$ & $1.44(1.41-1.46)$ \\
\hline 2 & $157820(11.0)$ & 141761 (89.8) & $2.90(2.85-2.95)$ & $1.56(1.52-1.59)$ & $1.63(1.60-1.67)$ \\
\hline 3 & $102251(7.1)$ & $93043(91.0)$ & 3.31 (3.24-3.39) & $1.70(1.65-1.74)$ & $1.83(1.78-1.88)$ \\
\hline 4 & $62598(4.4)$ & $57589(92.0)$ & $3.77(3.66-3.88)$ & $1.87(1.81-1.93)$ & $2.07(2.0-2.14)$ \\
\hline 5 & 54943 (3.8) & 51065 (92.9) & $4.32(4.18-4.46)$ & $2.12(2.04-2.2)$ & $2.44(2.35-2.54)$ \\
\hline \multicolumn{6}{|l|}{ Anxiolytics } \\
\hline No & 1393849 (97.2) & $1134650(81.4)$ & & & \\
\hline Yes & $39965(2.8)$ & 32267 (80.7) & $0.96(0.93-0.98)$ & - & $0.63(0.61-0.65)$ \\
\hline \multicolumn{6}{|l|}{ Hypnotics } \\
\hline No & $1391126(97.0)$ & $1130053(81.2)$ & & & \\
\hline Yes & 42688 (3.0) & 36864 (86.4) & $1.46(1.42-1.5)$ & - & $0.90(0.87-0.93)$ \\
\hline \multicolumn{6}{|l|}{ Antidepressants } \\
\hline No & 1197385 (83.5) & $964663(80.6)$ & & & \\
\hline Yes & 236429 (16.5) & 202254 (85.5) & $1.43(1.41-1.45)$ & - & $1.02(1.00-1.03)$ \\
\hline \multicolumn{6}{|l|}{ Antipsychotics } \\
\hline No & $1403757(97.9)$ & $1143196(81.4)$ & & & \\
\hline Yes & $30057(2.1)$ & 23721 (78.9) & $0.85(0.83-0.88)$ & - & $0.75(0.73-0.78)$ \\
\hline
\end{tabular}

\section{Discussion}

\section{Main findings}

Overall, approximately one in five adults in Northern Ireland were in receipt of a psychotropic medication, and this was a significant risk factor for lower uptake of the COVID-19 vaccines after adjustment for sociodemographic and health characteristics. There was evidence that the degree of disparity varied according to the type of medication, with the most extreme effects in those prescribed anxiolytics and antipsychotics. This trend persisted when we restricted the analyses to the under 40 years of age population, with more extreme odds ratios for those who were under 40 and in receipt of anxiolytics and hypnotics. We demonstrated that the higher prevalence of mental health conditions among people who live in more deprived areas did not explain the marked social gradients in vaccination uptake.

To our knowledge, this is the first nationwide study investigating variation in COVID-19 vaccination uptake among individuals with severe and common mental health disorders. Our whole-population study was conducted at a time when all adults were eligible, in contrast to two early reports. ${ }^{4,14}$ 


\section{Limitations}

The findings should be interpreted in the context of some methodological limitations that relate mainly to the use of psychotropic medications as proxy for mental health conditions, which was a methodological choice made as a result of the absence of individual-level primary and secondary care diagnostic data. We could have chosen to use admission to psychiatric in-patient services, which would have the benefit of high specificity but would have excluded the much larger proportion of psychiatric ill health that is managed in the community. Primary care data are not yet available for population-wide record-linkage research studies in Northern Ireland, although they too may lack precision. ${ }^{22}$ There is an established body of literature that uses psychotropic medications as an indicator of mental ill health in countries where access to primary care data are limited. For example, this approach was recently used in Manitoba, Canada, to indicate changes in mental health in children and adolescents during the COVID-19 pandemic. ${ }^{23}$ The benefits of using psychotropic medication as a proxy for mental ill health include population-wide coverage and reasonable face validity. The limitations include the potential for confounding by variation in access to health services and in the availability of non-pharmacological treatments. The use of psychotropic medications for relatively common non-psychiatric conditions is also a significant consideration. However, most of these caveats will lead to an underestimation of the size of the association between poor mental health and vaccine uptake rather than producing a spurious association. For example, a misclassification of patients with true mental ill health, either because they are being treated non-pharmacologically or because we do not have access to clozapine prescriptions which, in Northern Ireland, is only dispensed in secondary care, will tend to reduce the difference between psychiatric patients and the rest of the population. The misclassification bias arising from the inclusion of people who have been prescribed psychotropic medications for physical, rather than for mental, health problems is also more likely to lead to an underestimation of the association between poor mental health and vaccinations rather than being an explanation of it, because, as we have shown, people with physical health problems are more likely to receive vaccination and that this increases with the number of conditions.

Uncertainty about the true population size from administrative data is a potential source of bias, as people who have left the region but whose records cannot detect this will become part of the unvaccinated group who do not receive medicines. Weighting them to compensate for these extra records relies on the population estimate; if the population estimate was very inaccurate this could result in over- or underweighting people in the cohort who genuinely were in the region but had no contact with healthcare services.

Ethnicity is not recorded in NHAIS, and therefore we could not adjust for it. We excluded care home residents from our study, because this group received very specific interventions and prioritisation for the administration of COVID-19 vaccinations. The results of our study may not be generalisable to other countries, given the high prevalence of medication use for mental health in Northern Ireland; however, they are keeping in line with the few studies reporting lower uptake of COVID-19 vaccinations in people with severe mental illness. ${ }^{4,14}$

\section{Interpretation of our findings}

The causes of the reduced odds of COVID-19 vaccination associated with mental health conditions are probably complex and multifactorial and may differ according to the condition. The reduced odds of vaccination for people who were prescribed antipsychotics is consistent with an Israeli study that found those with schizophrenia had lower and later uptake of vaccination compared with the general population, ${ }^{14}$ and pre-publication findings from OpenSAFELY that reported lower uptake of COVID-19 vaccinations in those with severe mental illness early in the implementation of the vaccination programme. ${ }^{4}$ It is reported that individuals with severe mental illness are less likely to engage with health-promoting activities, which may be related to difficulties accessing services, fears about immunisation, as well as difficulties in following and applying government guidance. ${ }^{24}$ Regardless of the mechanism, the finding that people with mental health conditions, who are at high risk of symptomatic COVID-19 disease and experience higher rates of hospital admission and death, ${ }^{6,7}$ have reduced odds of being vaccinated, should prompt investigation to understand and remedy this situation.

Although we do not have evidence to allow us to infer the reason for it, the lower uptake in individuals who were prescribed anxiolytics might be related to fears about the safety of COVID-19 vaccines ${ }^{25}$ and perhaps avoidance behaviours that are common in anxiety disorders. ${ }^{26}$ Hypnotic medication was associated with reduced odds of vaccination, particularly pronounced in younger people. Reports suggest that sleep problems and hypnotic prescriptions have increased during the pandemic, particularly, in younger age groups. ${ }^{27,28}$ Sleep disturbances may interfere with executive cognitive functioning, ${ }^{29}$ although the side-effects of hypnotics, such as drowsiness, memory impairment and psychomotor limitations might contribute to lower uptake. On the other hand, there was no association between use of antidepressants and vaccine uptake for the population as a whole and a weak positive association in younger people. This is an unexpected finding in the context of the association between vaccination and the other psychotropic medications. It may be because of the complex interaction between depression and physical comorbidities that has been noted for influenza vaccination uptake. ${ }^{30}$ Investigation of this relationship in an independent cohort may yield further insights.

\section{Implications}

Vaccination is an important mechanism for reducing harm and disruption from the COVID-19 pandemic. Modelling suggests that vaccination improve the mental health of those most at risk of deaths or hospital admission by reducing levels of anxiety, although perhaps not for younger people who were at lower risk of harm from COVID-19. ${ }^{31}$ We urgently need innovative, evidence-based approaches to support people who have mental health conditions to be vaccinated. It is likely that this should include endorsement of vaccination from trusted sources and offering opportunistic or scheduled vaccination associated with routine mental healthcare contacts, such as through community psychiatric services and pharmacies. ${ }^{32}$ Monitoring and evaluation of such interventions will be crucial.

Siobhán Murphy (D, PhD, Centre for Public Health, Queen's University Belfast, UK; Dermot O'Reilly (D), MD, Centre for Public Health, Queen's University Belfast, UK; Rhiannon K. Owen (1D), PhD, Swansea University Medical School, Swansea University, UK; Ashley Akbari (D), MSc, Swansea University Medical School, Swansea University,

UK: Emily Lowthian (D), PhD, Swansea University Medical School, Swansea University,

UK; Stuart Bedston (ID), PhD, Population Data Science, Swansea University Medical

School, Swansea University, UK; Fatemeh Torabi (D, MSC, Swansea University Medical

School, Swansea University, UK; Jillian Beggs, Breathe, The Health Data Research Hub for Respiratory Health, UK; Antony Chuter, Breathe, The Health Data Research Hub for Respiratory Health, UK; Simon de Lusignan (D), MD, Nuffield Department of Primary Care Health Sciences, University of Oxford, UK; Richard Hobbs (iD), FMedSci, Nuffield Department of Primary Care Health Sciences, University of Oxford, UK:

Chris Robertson, PhD, Public Health Scotland, UK and Department of Mathematics and Statistics, University of Strathclyde, UK: Srinivasa Vittal Katikireddi (ID, PhD, MRC/CSO Social \& Public Health Sciences Unit, University of Glasgow, UK; Aziz Sheikh (D), MD, Usher Institute, University of Edinburgh, UK; Declan T. Bradley (D), PhD, Centre for Public Health, Queen's University Belfast, UK and Public Health Agency, UK

Correspondence: Declan T. Bradley. Email: declan.bradley@qub.ac.uk

First received 20 Oct 2021, final revision 26 Jan 2022, accepted 30 Jan 2022 


\section{Supplementary material}

To view supplementary material for this article, please visit https://doi.org/10.1192/bjp.2022.36.

\section{Data availability}

The data used in this study are available in the Honest Broker Service (HBS) within the Business Services Organisation Northern Ireland (BSO), but as restrictions apply, they are not publicly available. All proposals to use data are subject to review by an independent HBS Governance Board (HBSGB). Before any data can be accessed, approval must be given by the HBSGB. When access has been granted, it is gained through a privacy protecting safe haven and remote access system. HBS has established an application process to be followed by anyone who would like to access data, which can be found at https://hscbusiness.hscni.net/ services/2454.htm

\section{Acknowledgements}

The authors would like to acknowledge the help provided by the staff of the Honest Broker Service (HBS) within the Business Services Organisation Northern Ireland (BSO). The HBS is funded by the BSO and the Department of Health for Northern Ireland. The authors alone are responsible for the interpretation of the data and any views or opinions presented are solely those of the author and do not necessarily represent those of the BSO.

\section{Author contributions}

D O R. S.M and D T B conceived this study S.M drafted the protocol, which was contributed to by D.O.R. and D.T.B. Both S.M. and D. T.B. conducted all analyses and verified the data. S.M. to by D. .R. and D.T.B. Both S.M. and D.T.B. conducted all analyses and verified the data. S.M.
drafted the manuscript. All authors contributed to the study design and revised the manuscript for important intellectual content. All authors gave final approval of the version to be published.

\section{Funding}

UK Research and Innovation (Medical Research Council); Research and Innovation Industrial Strategy Challenge Fund; Health Data Research UK.

\section{Declaration of interest}

D.T.B. is jointly employed by Queen's University Belfast, the Public Health Agency and the Department of Health (Northern Ireland) and is currently, or has been, a member of COVID19 advisory groups in Northern Ireland and the UK including the UK Scientific Advisory Group for Emergencies and several of its subgroups. A.S. is a member of the Scottish Government Chief Medical Officer's COVID-19 Advisory Group and the New and Emerging Respiratory Virus Threats (NERVTAG) Risk Stratification Subgroup. C.R. is a member of the Scientific Pandemic Influenza Group on Modelling (SPI-M). All other authors report no conflicts of interest. S.V.K. acknowledges funding from a NRS Senior Clinical Fellowship (SCAF/15/02), the Medical Research Council (MC UU O0022/2) and the Scottish Government Chief Scientist Office (SPHSU17). R.K.O. is a member of the National Institute for Health and Care Excellence Technology Appraisal Committee.

\section{References}

1 Marmot M, Allen J, Goldblatt P, Herd E, Morrison J. Build Back Fairer: the COVID19 Marmot review. The pandemic, Socioeconomic and Health Inequalities in England. University College London, 2020 (http://www. instituteofhealthequity. org/resources-reports/build-back-fairer-the-covid-19-marmot-review/buildback-fairer-the-covid-19-marmot-review-full-report.pdf).

2 World Health Organization. The Impact of COVID-19 on Mental, Neurological and Substance use Services: Results of a Rapid Assessment. WHO, 2020 (https://www.who.int/publications/i/item/978924012455).

3 COVID-19 Mental Disorders Collaborators. Global prevalence and burden of depressive and anxiety disorders in 204 countries and territories in 2020 due to the COVID-19 pandemic. Lancet 2021; 398: 1700-12.

4 MacKenna B, Curtis HJ, Morton CE, Inglesby P, Walker AJ, Morley J, et al. Trends, regional variation, and clinical characteristics of COVID-19 vaccine recipients: a retrospective cohort study in 23.4 million patients using OpenSAFELY. MedRxiv [Preprint] 2021. Available from: https://doi.org/10. 1101/2021.01.25.21250356.

5 Office for National Statistics. Coronavirus and Vaccine Hesitancy by SubGroup, Great Britain: 13 January to 7 February 2021. Office for National Statistics, 2021 (https://www.ons.gov.uk/releases/coronavirusandvaccinehesitancybysubgroupgreatbritain13januaryto7february2021).

6 Wang Q, Xu R, Volkow ND. Increased risk of COVID-19 infection and mortality in people with mental disorders: analysis from electronic health records in the United States. World Psychiatry 2021; 20: 124-30.
7 Li L, Li F, Fortunati F, Krystal JH. Association of a prior psychiatric diagnosis with mortality among hospitalized patients with coronavirus disease 2019 (COVID-19) infection. JAMA Netw Open 2020; 3: e2023282.

8 De Hert M, Correll CU, Bobes J, Cetkovich-Bakmas M, Cohen DA, Asai I, et al. Physical illness in patients with severe mental disorders. I. prevalence, impact of medications and disparities in health care. World Psychiatry 2011; 10: 52-77.

9 John A, McGregor J, Jones I, Chim Lee S, Walters JTR, Owen MJ, et al. Premature mortality among people with severe mental illness - new evidence from linked primary care data. Schizophr Res 2018; 199: 154-62.

10 Glasheen C, Hedden SL, Forman-Hoffman VL, Colpe L. Cigarette smoking behaviors among adults with serious mental illness in a nationally representative sample. Ann Epidemiol 2014; 24: 776-80.

11 Ross E, Maguire A, Mairs A, Hall C, Donnelly MJC, O'Reilly DPJ. Disparities in breast cancer screening uptake for women with mental illness: a populationbased record linkage study. Am J Prev Med 2021; 60: e123-30.

12 De Picker L, Dias MC, Benros ME, Vai B, Branchi I, Benedetti F, et al. Severe mental illness and European COVID-19 vaccination strategies. Lancet Psychiatry 2021; 8: 356-9.

13 Jefsen $\mathrm{OH}$, Kølbæk P, Gil Y, Speed M, Dinesen PT, Sønderskov KM, et al. COVID19 vaccine willingness amongst patients with mental illness compared with the general population. Acta Neuropsychiatr 2021; 1: 1-4.

14 Bitan DT, Kridin K, Cohen AD, Weinstein O. COVID-19 hospitalisation, mortality, vaccination, and postvaccination trends among people with schizophrenia in Israel: a longitudinal cohort study. Lancet Psychiatry 2021; 8: $901-8$.

15 O'Neill S, Heenan D, Betts J. Review of Mental Health Policies in Northern Ireland: Making Parity a Reality. Ulster University, 2019 (https://www.ulster.ac. uk/_data/assets/pdf_file/0004/452155/Final-Draft-Mental-Health-Reviewweb.pdf).

16 UK Government. Vaccinations in United Kingdom. UK Government, 2021 (https://coronavirus.data.gov.uk/details/vaccinations).

17 UK Government. Adult Social Care Monthly Statistics England. UK Government, 2021 (https://www.gov.uk/government/statistics/adult-social-care-in-england-monthly-statistics-june-2021/adult-social-care-monthly-statistics-england-june-2021).

18 Burton JK, Guthrie B. Identifying who lives in a care home-a challenge to be conquered.identifying who lives in a care home-a challenge to be conquered. Age Ageing 2018; 47: 322-3

19 Northern Ireland Statistics and Research Agency. Northern Ireland Multiple Deprivation Measure. Northern Ireland Statistics and Research Agency, 2017 (https://www.nisra.gov.uk/statistics/deprivation/northern-ireland-multipledeprivation-measure-2017-nimdm2017).

20 Henderson DA, Atherton I, McCowan C, Mercer SW, Bailey N. Linkage of national health and social care data: a cross-sectional study of multimorbidity and social care use in people aged over 65 years in Scotland. Age Ageing 2021: 50: $176-82$.

21 Vasileiou $E$, Simpson $C R$, Shi T, Kerr S, Agrawal $U$, Akbari A et al. Interim findings from first-dose mass COVID-19 vaccination roll-out and COVID-19 hospital admissions in Scotland: a national prospective cohort study. Lancet 2021; 397: 1646-57.

22 Mitchell A, Vaze A, Rao S. Clinical diagnosis of depression in primary care: a meta-analysis. Lancet 2009; 374: 609-19.

23 Leong C, Katz LY, Bolton JM, Enns MW, Delaney J, Tan Q, et al. Psychotropic drug use in children and adolescents before and during the COVID-19 pandemic. JAMA Pediatr 4 Jan 2022 [Epub ahead of print]. Available from: https://doi.org/10.1001/jamapediatrics.2021.5634.

24 Miles LW, Williams N, Luthy KE, Eden L. Adult vaccination rates in the mentally ill population: an outpatient improvement project. J Am Psychiatr Nurses Assoc 2020; 26: 172-80.

25 De Figueiredo A, Simas C, Karafillakis E, Paterson P, Larson HJ. Mapping global trends in vaccine confidence and investigating barriers to vaccine uptake: a large-scale retrospective temporal modelling study. Lancet 2020; 396 898-908.

26 Heimberg RG, Turk CL, Mennin DS. Generalized Anxiety Disorder: Advances in Research and Practice. Guilford Press, 2004.

27 Beck F, Léger D, Fressard L, Peretti-Watel P, Verger P, Coconel Group. Covid-19 health crisis and lockdown associated with high level of sleep complaints and hypnotic uptake at the population level. J Sleep Res 2021; 30: e13119.

28 Keller MS, Kiefer E, Campbell S, Bradley K, Mashburn R, Bawa M, et al. Sustained increase of sedative-hypnotic prescribing during the COVID-19 pandemic in a large Urban health system: an observational study. J Gen Intern Med 2021; 13: 1-3.

29 Ballesio A, Aquino MRJV, Kyle SD, Ferlazzo F, Lombardo C. Executive functions in insomnia disorder: a systematic review and exploratory meta-analysis. Front Psychol 2019; 10: 101 
30 Lawrence T, Zubatsky M, Meyer D. The association between mental health diagnoses and influenza vaccine receipt among older primary care patients. Psychol Health Med 2020; 25: 1083-93.

31 Chaudhuri K, Howley P. The impact of Covid-19 vaccination on mental health. SSRN 2021. Available from: http://dx.doi.org/10.2139/ssrn.3906235.
32 Warren N, Kisely S, Siskind D. Maximizing the Uptake of a COVID-19 vaccine in people with severe mental illness: a public health priority. JAMA Psychiatry 2021; 78: 589-90.

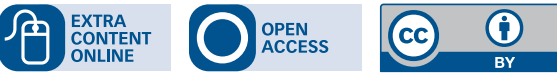

\section{Extra poems}

\section{Bipolar villanelle and Lithium}

\section{Rebecca Lawrence (iD}

Bipolar villanelle

Such rhapsody is mine at last Yet not for long, my mind can tell, Pain and misery will hold me fast.

Cruel melancholia is past, the grinding sorrow, darkened hell

such rhapsody is mine at last.

But still I know with all the haste Of joy, that change will sound a knell, Pain and misery will hold me fast.

This drama has me quite outclassed, despite the times when all is well such rhapsody is mine at last.

Soon complacent, then aghast By that returning vicious hell Pain and misery will hold me fast.

My life would clearly be a blast Without bipolar's nasty spell.

Such rhapsody is mine at last Pain and misery will hold me fast.

Lithium

Lithium!

Element and traitor

What do you do to me?

but soothe my tongue,

belie my mind.

I see you

stretching through the years

at times abandoned

white chalk hard

What are you?

A two-faced mask

stealing feelings

You take my happiness

and laugh at it

through tears.

Metal, we are one

You have my joys, my sorrows,

you make the night kind

and the day that follows

unremarkable. 